\title{
The CMS Silicon Pixel Detector for HL-LHC
}

\section{Georg Steinbrück*}

Institute for Experimental Physics

Hamburg University

Luruper Chaussee 149

22761 Hamburg, Germany

E-mail: georg.steinbrueck@desy.de

\section{for the CMS collaboration}

The LHC is planning an upgrade program which will bring the luminosity to about $5 \times 10^{34} \mathrm{~cm}^{-2} \mathrm{~s}^{-1}$ in 2026 , with the goal of an integrated luminosity of $3000 \mathrm{fb}^{-1}$ by the end of 2037. This High Luminosity scenario, HL-LHC, will present new challenges of higher data rates and increased radiation. To maintain its physics potential in this harsh environment, the CMS detector will undergo a major upgrade program known as the Phase II upgrade. The new Phase II pixel detector will require a high bandwidth readout system and highly radiation tolerant sensors and on-detector ASICs. Several technologies for the sensors are being studied. Serial powering schemes are under consideration to accommodate significant constraints on the system. These prospective designs, as well as new layout geometries that include very forward pixel discs with acceptance extended up to $|\eta|<4$, are presented together with performance estimates.

The 25th International workshop on vertex detectors

September 26-30, 2016

La Biodola, Isola d'Elba, ITALY

*Speaker. 


\section{Introduction}

During the high-luminosity phase of the LHC (HL-LHC), the machine is foreseen to deliver an integrated luminosity of $\mathscr{L}_{\text {int }}=3000 \mathrm{fb}^{-1}$ in the decade 2026-2037 at a center-of-mass energy of $14 \mathrm{TeV}$. This will be achieved by increasing the instantaneous luminosity up to 5 or even $7.5 \times 10^{34} \mathrm{~cm}^{-2} \mathrm{~s}^{-1}$ in so-called ultimate scenarios. To maintain and even improve its physics potential in this harsh environment, CMS will undergo a major upgrade during Long Shutdown 3 (LS3), completely replacing the tracking system $[1,2]$. The pixel detector will be based on finepitch hybrid pixel sensors. While thin planar silicon sensors will be used throughout most of the detector, 3D silicon sensors are being considered as an option for the innermost layer.

A map of the expected fluence as a function of the radius ${ }^{1}$ at the end of the HL-LHC running period is shown in Fig. 1. At the inner pixel radius of $3 \mathrm{~cm}$, a fluence of particles corresponding to a non-ionizing energy loss (NIEL) of $\Phi=2 \times 10^{16} 1 \mathrm{MeV} \mathrm{n}$ eq $/ \mathrm{cm}^{2}$ will be reached. The corresponding total ionizing dose (TID) will be about $10 \mathrm{MGy}$. The peak instantaneous luminosity with a bunch-crossing separation of $25 \mathrm{~ns}$ will lead to an average number of simultaneous inelastic collisions per crossing (pileup) of 200 which in the innermost region translates into a rate of charged particles of about $750 \mathrm{MHz} / \mathrm{cm}^{2}$ or equivalently into a hit rate of $3 \mathrm{GHz} / \mathrm{cm}^{2}$.

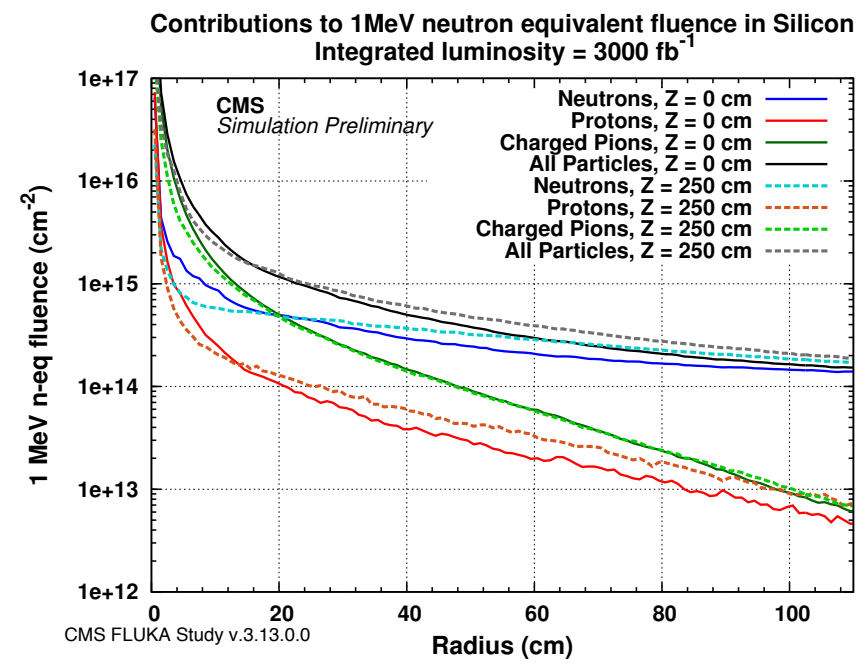

Figure 1: Fluence versus radius expected at CMS for an integrated luminosity of $3000 \mathrm{fb}^{-1}$ at HL-LHC [3].

\section{Detector layout}

A baseline design (Fig. 2) for the CMS tracking system for the HL-LHC has been developed and will be published in the Tracker Technical Design Report (TDR) in 2017. The layout of this

\footnotetext{
${ }^{1}$ The coordinate system adopted by CMS has the origin centered at the nominal collision point inside the experiment, the $y$-axis pointing vertically upward, and the $x$-axis pointing radially inward toward the center of the LHC. Thus, the $z$-axis points along the beam direction toward the Jura mountains from LHC Point 5. The azimuthal angle $\phi$ is measured from the $x$-axis in the $x-y$ plane and the radial coordinate in this plane is denoted by $r$. The polar angle $\theta$ is measured from the $z$-axis. Pseudorapidity is defined as $\eta=-\ln \tan (\theta / 2)$.
} 
tracker has been developed using a dedicated software package, tkLayout [4][5], which estimates the resolutions of track parameters based on the position of the sensing elements, the resolutions of the coordinates measured at each plane and the effects of inactive material and services for each configuration under investigation. The tracker is divided into two parts: the pixel detector and the Outer Tracker (OT) detector. The OT consists of modules with two silicon sensors glued backto-back (2S and PS modules) for on-module transverse momentum estimation used in the level 1 trigger. The pixel detector consists of a barrel and a forward region (Fig. 3). The barrel section of

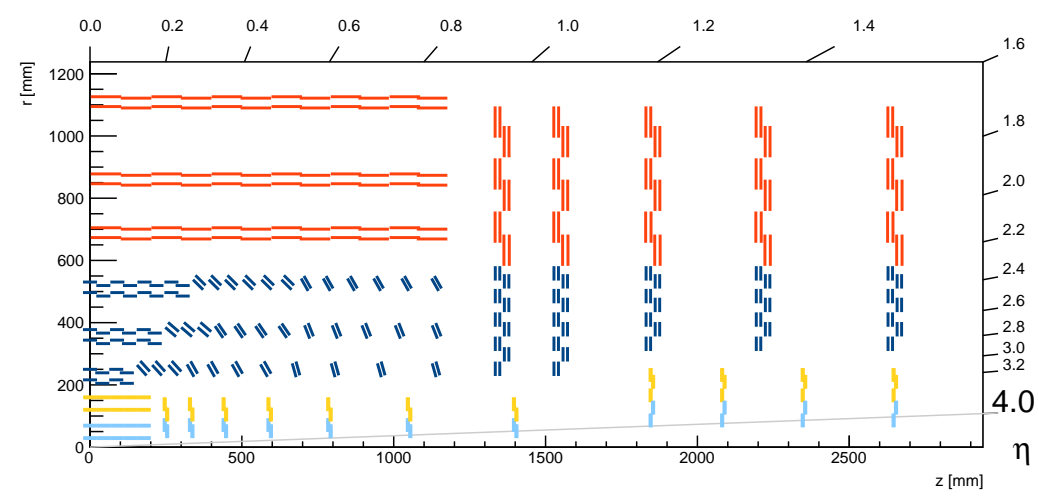

Figure 2: Layout of the CMS tracking system for the HL-LHC. Outer Tracker layers with modules consisting of two back-to-back strip sensors ( $2 \mathrm{~S}$ modules) are shown in red $(r>60 \mathrm{~cm})$, layers with modules consisting of a macro-pixel and a strip sensor (PS modules) are shown in blue $(20 \mathrm{~cm}<r<60 \mathrm{~cm})$. The pixel detector, depicted in light blue and yellow, consists of four barrel layers $(r<20 \mathrm{~cm})$ and two sets of 11 discs.

the pixel detector is composed of four cylindrical layers extending from $r=2.9 \mathrm{~cm}$ to $r=16 \mathrm{~cm}$. Compared with the Phase I detector [6], the length is reduced to $\left|z_{\max }\right|=20 \mathrm{~cm}$ to give more room to the extended forward pixel system. It consists of two sets of 11 discs located on both sides of the barrel. To guarantee a uniform coverage in pseudorapidity $\eta$, the last four discs start at a larger radius, $r=6.4 \mathrm{~cm}$, and extend up to $r=25 \mathrm{~cm}$. To enable the installation of the central part

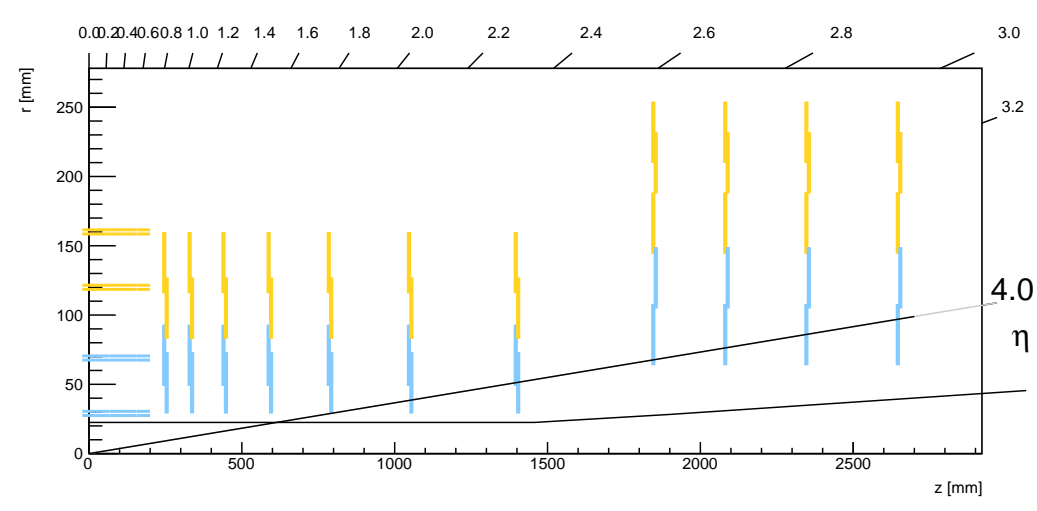

Figure 3: Layout of the CMS pixel detector for the HL-LHC. Modules consisting of $1 \times 2$ readout chips are depicted in light blue, modules with $2 \times 2$ readout chips are drawn in yellow. 
of the detector with the beampipe in place, clearance is added at the far edge of the detector by introducing a step in the envelope between the pixel detector and the Outer Tracker from $r=20 \mathrm{~cm}$ to $r=30 \mathrm{~cm}$ at $z=160 \mathrm{~cm}$. The pixel detector covers a total area of $4.5 \mathrm{~m}^{2}$.

Two different pixel geometries are studied for a total pixel area of $2500 \mu \mathrm{m}^{2}$ : rectangular pixels $\left(25 \times 100 \mu \mathrm{m}^{2}\right)$ and square pixels $\left(50 \times 50 \mu \mathrm{m}^{2}\right)$. To compare the impact of pixel geometry on hit and track resolutions, a Monte Carlo study was performed using high transverse momentum single muon tracks processed through the CMS detector simulation and offline reconstruction software. The spatial resolution was determined using the known position of the particle from the Monte Carlo truth. In this study, two sensor thicknesses were considered, $100 \mu \mathrm{m}$ and $150 \mu \mathrm{m}$, as well as three preamplifier thresholds for the signal in a single pixel cell (1000 e, $1500 \mathrm{e}$ and $2000 \mathrm{e}$ ), Fig. 4 [7]. As a reference, the readout chip (ROC) under development for the HL-LHC is planned to feature four bit pixel charge information and sparsified readout with a design in-time threshold around $1000 \mathrm{e}$. The resolution in the transverse plane is, in general, better for rectangular pixels since their short side is oriented in the $r \phi$ direction. In $z$, the resolution is typically better for square
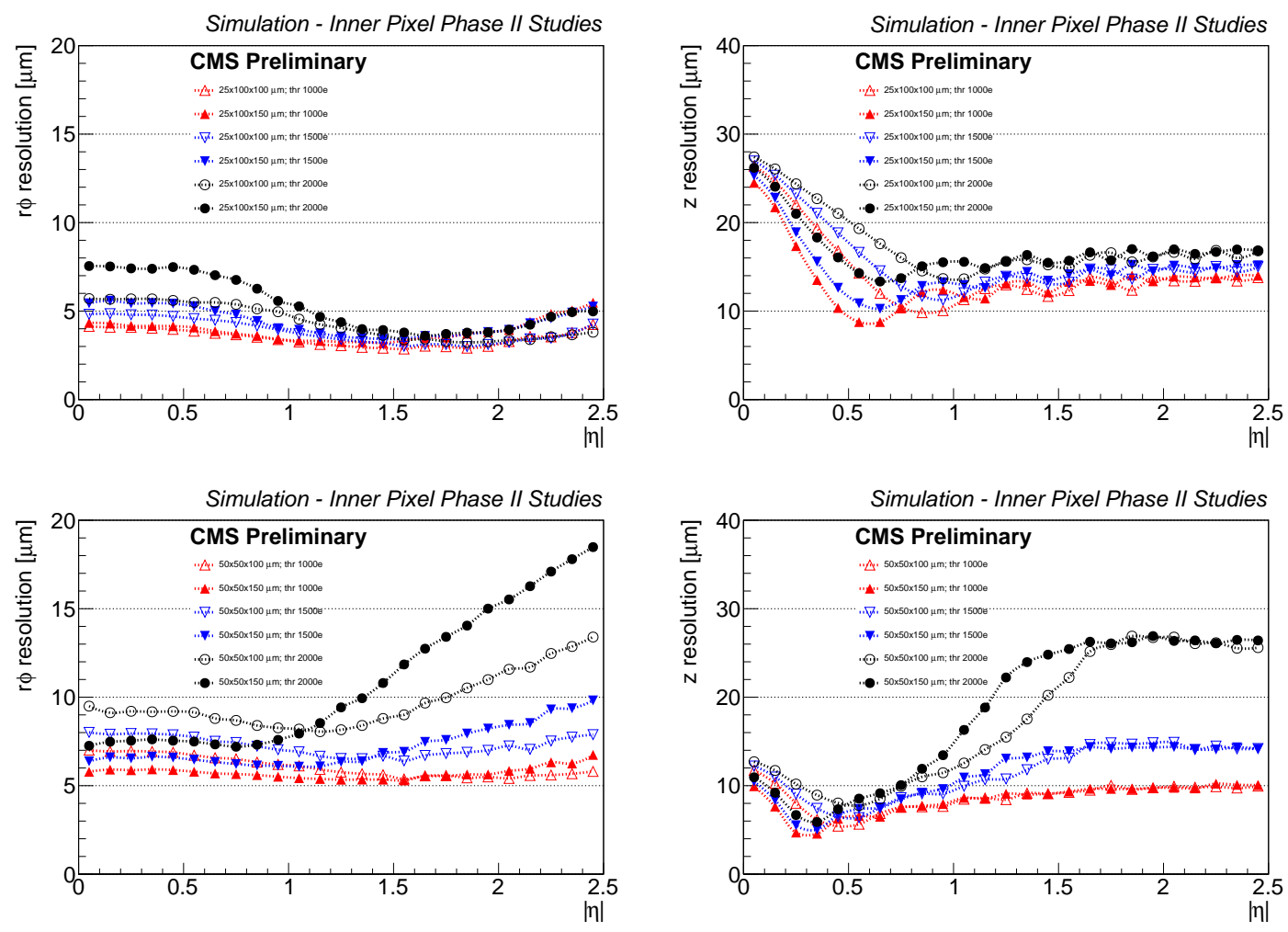

Figure 4: Hit resolution in $r \phi$ (left) and $z$ (right) as a function of $\eta$ for small pitch pixels for different thresholds and sensor thicknesses for the first layer of the barrel. This study is based on high transverse momentum single muon Monte Carlo tracks generated and processed through the CMS detector simulation and reconstruction software. Red: threshold=1000 e, blue: threshold=1500 e, black: threshold=2000 e; open symbols: $100 \mu \mathrm{m}$ sensor thickness, filled symbols: $150 \mu \mathrm{m}$ sensor thickness. Upper row: $25 \times 100 \mu \mathrm{m}^{2}$ pixel size. Lower row: $50 \times 50 \mu \mathrm{m}^{2}$ pixel size. The results do not include the effects of radiation damage. 
pixels. However, for square pixels the resolution strongly deteriorates at large $\eta$ for thicker sensors and high thresholds. This is due to the breakage of clusters which contain many pixels due to the combination of large path lengths and a small pitch in $z$-direction. An improved cluster algorithm would help to increase the resolution for these cases. This study did not take into account the effects of radiation damage, but the effects of a lower signal due to trapping are similar to a larger threshold. While strong quantitative statements can only be deduced when the reduced signal and increased noise after irradiation, as well as pile-up and tracking in a dense environment are taken into account, this study supports the preference for a rectangular pixel geometry.

\section{Pixel sensor R\&D}

CMS has embarked on a broad R\&D program to develop fine-pitch pixel sensors for the HLLHC. The outer layers of the pixel detector will be equipped with planar silicon sensors, likely produced on 6 inch $\mathrm{n}^{+}$-in-p wafers. A major challenge is the severe radiation the sensors are subjected to. Figure 5 shows the collected charge, measured using an infrared laser setup, for irradiated pad diodes with an active thickness of $200 \mu \mathrm{m}$ as a function of fluence for a bias voltage of $900 \mathrm{~V}$. At the highest $1 \mathrm{MeV}$ neutron equivalent fluence considered in this study $\left(1.3 \times 10^{16} \mathrm{n}_{\mathrm{eq}} / \mathrm{cm}^{2}\right), 5000$ electrons are collected for this rather high bias voltage. Figure 6 shows the collected charge as a func-

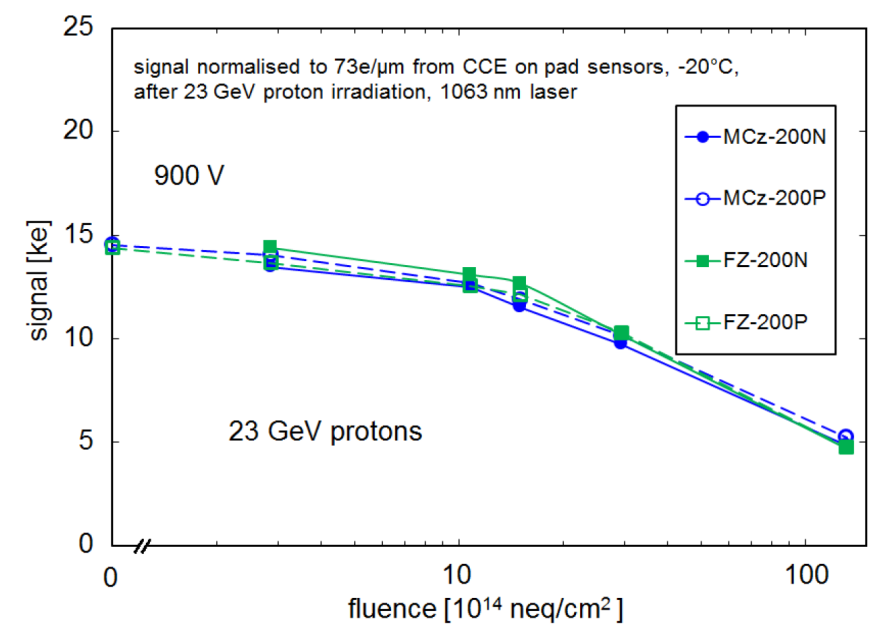

Figure 5: Collected charge for pad diodes at $900 \mathrm{~V}$ as a function of fluence for irradiation with $23 \mathrm{GeV}$ protons and neutrons, after 10 minutes annealing at $60^{\circ} \mathrm{C}[8]$. Both $\mathrm{n}^{+}$-in-p $(\mathrm{P})$ and $\mathrm{p}^{+}$-in- $\mathrm{n}(\mathrm{N})$ diodes were measured. The bulk material was either magnetic Czochralski (MCz) or float zone (FZ) silicon.

tion of bias voltage for mini strip sensors irradiated to fluences between 0 and $1.3 \times 10^{16} \mathrm{n}_{\text {eq }} / \mathrm{cm}^{2}$. This study was done with $200 \mu \mathrm{m}$ thick magnetic Czochralski (MCz) sensors and $100 \mu \mathrm{m}$ thick epitaxial (Epi) sensors. At $1.3 \times 10^{16} \mathrm{n}_{\mathrm{eq}} / \mathrm{cm}^{2}$, the $100 \mu \mathrm{m}$ thick sensor collects about the same charge as the $200 \mu \mathrm{m}$ thick sensor. At high fluences, severe trapping reduces the effective drift distance of the charge carriers so much that thicker silicon looses its advantage. The active thickness of pixel sensors for the HL-LHC upgrade of CMS will therefore likely be around $150 \mu \mathrm{m}$. Charge collection studies will be repeated with pixel sensors once fine-pitch pixel readout chips are avail- 
able. While the general trend will be similar, differences are expected in the charge collected on each pixel due to the different weighting field.

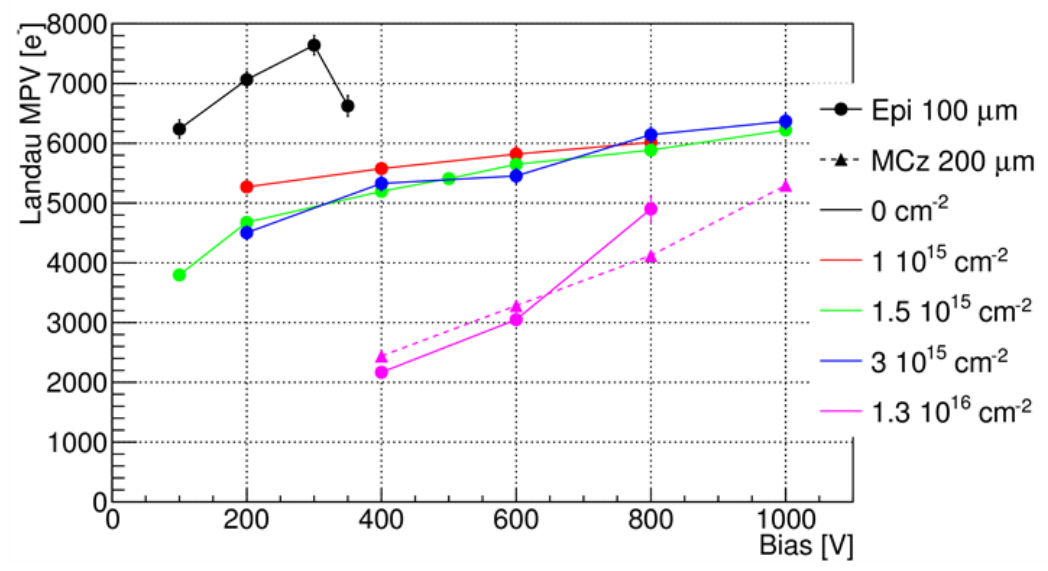

Figure 6: Collected charge as a function of bias voltage for irradiated $200 \mu \mathrm{m}$ thick magnetic Czochralski $(\mathrm{MCz} 200 \mu \mathrm{m})$ and $100 \mu \mathrm{m}$ thick epitaxial strip sensors (Epi $100 \mu \mathrm{m})$ [9]. The measurements were performed in an electron testbeam at DESY (momentum in the range of 3.5 to $5 \mathrm{GeV}$ ). The sensors were irradiated with $23 \mathrm{GeV}$ protons at the CERN PS. Fluences are given in units $1 \mathrm{MeV} \mathrm{n}$ eq $/ \mathrm{cm}^{2}$.

CMS has initiated several R\&D submissions to study design options for thin fine-pitch sensors, summarized in Table 1. These submissions have in common that they contain sensors for a variety of existing and future pixel readout chips, such as PSI46dig, RD53A [10, 11], ROC4sens (an R\&D chip developed at PSI, Switzerland), FCP130 (an R\&D chip under development at Fermilab), and FE-I4 (mainly for serial powering studies). The sensors for the PSI46dig readout chip typically

Table 1: Summary of R\&D submissions for the Phase II upgrade of the CMS pixel detector. The key parameters and main goals of the three planar and two 3D submissions are listed.

\begin{tabular}{|l|l|l|}
\hline Producer & Wafer characteristics & Goals of the submission \\
\hline Hamamatsu Photonics & $\begin{array}{l}n^{+} \text {-in- } p \\
\text { active thickness: } 150 \mu \mathrm{m}\end{array}$ & $\begin{array}{l}\text { pixel geometry: rectangular versus square } \\
\text { common } p \text {-stop vs. } p \text {-spray isolation } \\
\text { bias scheme for small pixels (no bias, common punch-through, poly-Si) } \\
\text { metal overhang to mitigate E-field }\end{array}$ \\
FBK & $\begin{array}{l}n^{+} \text {-in- } p \\
\text { active thickness: } 100-130 \mu \mathrm{m}\end{array}$ & $\begin{array}{l}\text { punch-through biasing scheme } \\
\text { spark protection using BCB } \\
\text { different wafer thinning procedures }\end{array}$ \\
Sintef & $\begin{array}{l}n^{+} \text {-in- } n \\
\text { active thickness: } 300 \mu \mathrm{m}\end{array}$ & $\begin{array}{l}\text { slim edge (active area } 210 \mu \mathrm{m} \text { from dicing edge) } \\
\text { slim pixels }\left(25 \times 600 \mu \mathrm{m}^{2}\right)\end{array}$ \\
\hline FBK 3D & $\begin{array}{l}n^{+} \text {-in- } p \\
\text { active thickness: } 100-130 \mu \mathrm{m} \\
\text { single sided DRIE process }\end{array}$ & small pixel sizes on thin substrate \\
& $\begin{array}{l}n^{+} \text {-in- } p \\
\text { active thickness: } 230 \mu \mathrm{m} \\
\text { double sided process }\end{array}$ & $\begin{array}{l}\text { small aspect ratios: } 8 \mu \mathrm{m} \text { holes in } 230 \mu \mathrm{m} \text { Si } \\
\text { slim edges }(100 \text { and } 200 \mu \mathrm{m})\end{array}$ \\
\hline
\end{tabular}


contain a few small pixels $\left(25 \times 100 \mu \mathrm{m}^{2}\right.$ and $\left.50 \times 50 \mu \mathrm{m}^{2}\right)$ routed to larger readout pixels $(100 \times 150$ $\mu \mathrm{m}^{2}$ ), enabling the study of small pitch pixels before a dedicated chip is ready. However, most of the studies will be done using the small pitch chips RD53A and ROC4sens. As an example, Fig. 7 shows the wafer layout for the submission with Hamamatsu Photonics (HPK). The aim of these

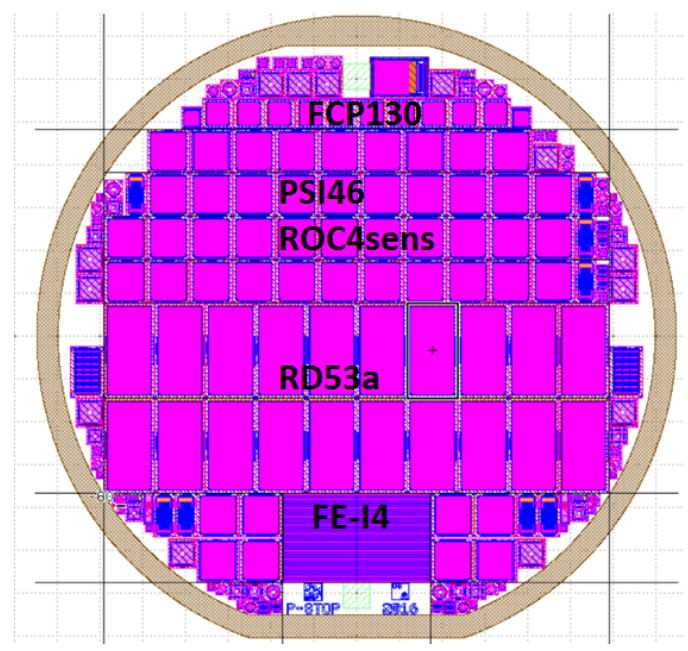

Figure 7: Layout of the p-stop wafer for the R\&D submission with HPK. Sensors for a variety of readout chips are visible as well as dedicated test structures and pad diodes.

submissions is to evaluate and optimize design options for small pixel sizes where some of the typical choices might not work anymore due to space constraints and conflicts with the design rules of the producers. The main concerns are listed below:

- Pixel bias schemes to test sensors before bump bonding. Considered options are punch through biasing common to several adjacent pixels instead of individual bias dots, polysilicon resistors, or no biasing scheme at all.

- Pixel isolation: $\mathrm{p}$-stop versus $\mathrm{p}$-spray isolation. To save space, $\mathrm{p}$-stop implants common to adjacent pixels instead of individual ones have been implemented.

- Metal overhangs to mitigate large electric fields at the pixel implant edges.

Sensors with pixel sizes of $25 \times 100 \mu \mathrm{m}^{2}$ and $50 \times 50 \mu \mathrm{m}^{2}$ have been implemented in various design variants and will be evaluated for radiation hardness, spatial resolution and charge collection/ pixel hit efficiency in testbeams.

While most of the CMS pixel detector will be based on thin, fine-pitch planar pixel sensors, for the innermost layer both planar and 3D sensors are being considered. The advantage of sensors built in 3D technology is that the sensor thickness is decoupled from the charge collection distance by introducing column electrodes. While more initial charge carriers are generated in a thicker sensor, a short drift distance is advantageous for sensors subjected to large fluences when charge particle trapping becomes important. Such sensors are already in use in the ATLAS Insertable B-Layer (IBL) [12] detector. R\&D programs with FBK (Italy) and CNM (Spain) are ongoing to develop fine-pitch $3 \mathrm{D}$ sensors suitable for the first layer. Both producers have successfully produced and 
tested 3D sensors for the existing PSI46dig and FE-I4 readout chips. Recently completed submissions include small pixel sensors for the aforementioned R\&D chips and are ready for bump bonding and testing as soon as the chips will be available.

\section{The pixel readout chip}

Operation under HL-LHC conditions imposes strong demands on the pixel readout chip for the Phase II upgrade. These are:

- Radiation tolerance up to $10 \mathrm{MGy}$ of TID. This requirement is reduced to $5 \mathrm{MGy}$ if CMS decides to replace the inner layer of the pixel detector once during the HL-LHC;

- Low noise $(\sim 1000$ e) for efficient hit finding even with small signals due to the reduced thickness of the sensors and irradiation damage;

- A deep readout buffer and fast readout rate to comply with the $12.5 \mu$ s latency and $750 \mathrm{kHz}$ readout rate required by the CMS experiment.

- A pixel area of only $2500 \mu \mathrm{m}^{2}$.

The baseline is to adopt a ROC designed in $65 \mathrm{~nm}$ technology based on the developments within the RD53 collaboration $[10,11]$. The size of the ROC will be around $2 \times 2 \mathrm{~cm}^{2}$.

\section{Serial powering}

The total power required by the pixel detector is driven by the total number of ROCs (about 12000) and by the ROC power consumption (about $1 \mathrm{~W} / \mathrm{cm}^{2}$ for a $2 \times 2 \mathrm{~cm}^{2}$ chip): It will be around $40-50 \mathrm{~kW}$. A powering scheme based on DC-DC converters, similar to the one designed for the Phase I detector, is not feasible because of the limited radiation tolerance of the ASICs, and because of space constraints for placing these components close to the ROC. Therefore, an across-module serial powering scheme, where the power is supplied sequentially to clusters of chips and then distributed in parallel within each cluster, is currently under investigation. This implementation will allow to keep the material budget of the power cables below $2 \mathrm{~kg}$. Central to the serial powering approach is the shunt LDO (low dropout regulator) which, integrated into the ROC, provides regulated voltages and shunts the excess current. This circuit was developed for the ATLAS FE-I4 chip and is currently being ported by RD53. A schematic of this approach can be seen in Fig. 8.

\section{Modularity and modules}

The electrical-to-optical conversion of the readout and control signals will occur inside a lowpower GigaBit Tranceiver card (LP-GBT) located at $r \sim 20 \mathrm{~cm}$ as currently no optoelectronic device can tolerate the level of radiation foreseen in the innermost layer of the pixel detector. The modularity of the detector in terms of ROCs is then defined by matching the input specifications of 


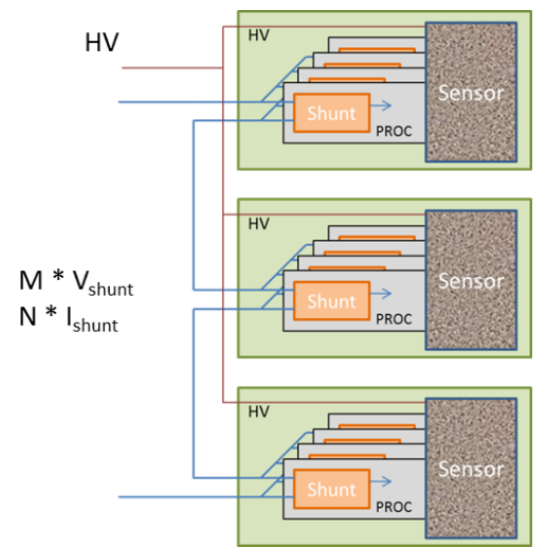

Figure 8: Serial power distribution with $\mathrm{N}$ pixel chips in parallel on the module and M modules connected in series.

the LP-GBT (7 input links with a bandwidth of $1.28 \mathrm{Gbit} / \mathrm{s}$ each) with the output rate of a module. The latter depends on the size of the ROC and on its position in the detector.

It is foreseen to minimize the number of different module types. Currently, only two pixel module types are considered to be used both in the barrel and the forward detector, consisting of $2 \times 1$ and $2 \times 2$ ROCs, respectively. A possible configuration for a module in the innermost layer of the barrel is shown in Fig. 9.

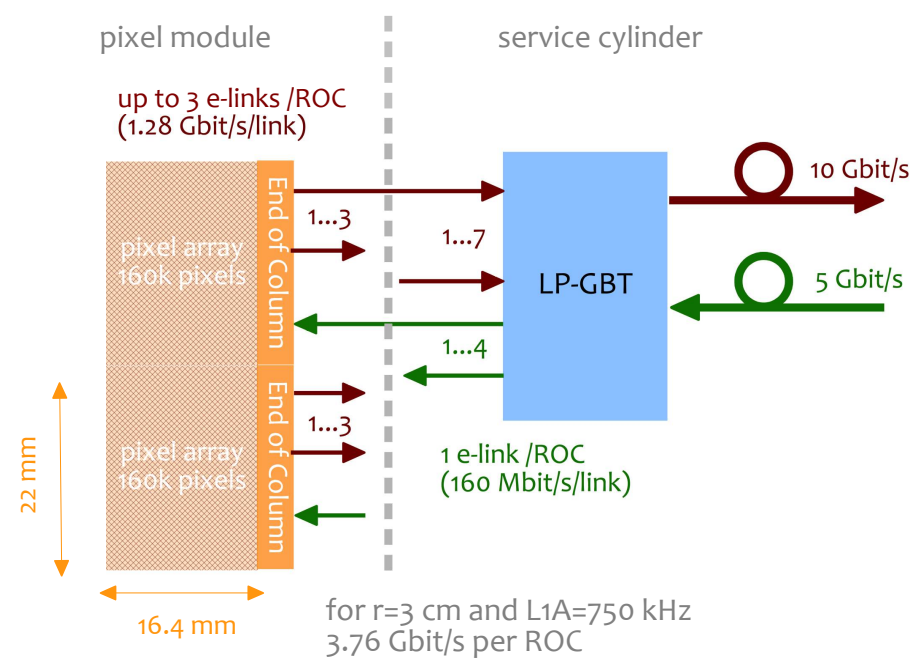

Figure 9: Possible arrangement for the readout of a module in the innermost layer of the barrel: a module made of $2 \times 1$ ROCs is read out by one LP-GBT chip. In the figure one of the electrical link (e-link) inputs is left unconnected. 


\section{Conclusion}

CMS will replace its entire tracking system for the HL-LHC. The new pixel detector will allow efficient tracking and vertexing under very demanding conditions with an angular coverage extended up to $|\eta|=4$. The requirements on the pixel detector are very high in terms of radiation hardness and hit rates. CMS has therefore initiated a vigorous R\&D program to develop an adequate pixel detector for this upgrade. Submissions for thin, fine-pitch pixel sensor prototypes have been initiated with various vendors and will be evaluated in the near future, using a variety of existing and new readout chips. These submissions comprise both planar pixel sensors and 3D sensors which are considered as an option for the first layer of the detector.

A radiation tolerant, fine-pitch, low noise readout chip is being designed by the CERN RD53 collaboration. A serial powering scheme and new high bandwidth readout architecture for the pixel detector is under development.

\section{References}

[1] CMS Collaboration, Technical Proposal for the Phase-II Upgrade of the Compact Muon Solenoid, CERN-LHCC-2015-10, LHCC-P-008, CMS-TDR-15-02, ISBN 978-92-9083-417-5 (2015).

[2] CMS Collaboration, CMS Phase II Upgrade Scope Document, CERN-LHCC-2015-019; LHCC-G-165 (2015).

[3] http://cds.cern.ch/record/2039908

[4] S. Mersi, D. Abbaneo, N. De Maio and G. Hall, Software package for the characterization of tracker layouts, in Astroparticle, particle, space physics and detectors for physics applications 7, World Scientific Publishing (2012).

[5] G. Bianchi, tkLayout: a design tool for innovative silicon tracking detectors, J. Instrum. 9 (2014) C03054 (2014).

[6] CMS Collaboration, CMS Technical Design Report for the Pixel Detector Upgrade, CERN-LHCC-2012-16, CMS-TDR-011(2012).

[7] G. Steinbrïck for the CMS Collaboration, Small pitch pixel sensors for the CMS Phase II upgrade, Proceedings of the Nuclear Science Symposium and Medical Imaging Conference (NSS/MIC), 2015 IEEE, CMSCR-2015/312.

[8] T. Poehlsen, Charge Losses in Silicon Sensors and Electric-Field Studies at the Si-SiO2 Interface, $\mathrm{PhD}$ Thesis at the University of Hamburg, DESY-THESIS-2013-025 (2013).

[9] M. Centis Vignali, Silicon Sensors for the Upgrades of the CMS Pixel Detector, PhD Thesis at the University of Hamburg, DESY-THESIS-2015-052 (2015).

[10] J. Chistiansen, M. Garcia-Sciveres, RD Collaboration proposal: Development of pixel readout integrated circuits for extreme rate and radiation, CERN-LHCC-2013-008; LHCC-P-006 (2013).

[11] RD53 Collaboration, RD53A Integrated Circuit Specifications, CERN-RD53-PUB-15-001 (2015).

[12] ATLAS Collaboration, ATLAS Insertable B-Layer Technical Design Report, CERN-LHCC-2010-13, ATLAS TDR 19, (2010). 